\title{
Fuzzy Classification and Implementation Methods for Tugboat Main Engine Fault
}

\author{
Xiaohui Xu , Guangchen Bai \\ School of Automation, Beijing University of Posts and Telecommunications, 100876 Beijing, China
}

\begin{abstract}
The establishment of classification index system of fault in tugboat main engine was briefly discussed first. After that, the fuzzy method and procedures used in fault classification were introduced. Several implementation techniques of the fuzzy classification were explored including the knowledge expression, the hierarchical structure of knowledge library, the pre-processor and the forward inference engine. Some specific fault records of tugboat main engine were analyzed and classified for certification. And results prove that fuzzy classification has certain reference value and improves the working efficiency of tugboat personnel in equipment maintenance and management.
\end{abstract}

\section{Introduction}

As an important auxiliary ship for towing larger vessels in harbor, tugboat has the characteristics of flexible operation and being highly maneuverable. However, at the same time it makes the load of tugboat main engine change dramatically, where the working condition is extremely severe. When it comes to tugboat fault diagnosis, personnel often relate to unquantifiable concepts such as relatively low pressure, insufficient flow and excessive temperature rise, which have not definite boundaries, namely "fuzzy concept". In addition, a fault may result in a variety of phenomena, especially for the heavy complicated engineering equipment integrated with electromechanical and hydraulic system. Deterministic factors and random factors being staggered together, tugboat main engine fault has the distinguishing feature of changing gradually and concealment[1]. Therefore, it's necessary to explore the classification index system of tugboat fault. By researching the classification method and its implement techniques, tugboat fault can be analyzed and classified, laying the foundation for the subsequent research. Tugboat fault classification will help personnel further identify the exact location and cause of fault, predict the trend of fault and potential danger, and thus determine the appropriate measures to be taken.

\section{Fault classification index system}

Tugboat works in the harbor, which is highly maneuverable and has high work intensity, therefore tugboat main engine adopt four-stroke medium speed diesel engine, with the advantages of compact structure and high power. Tugboat main engine workload's varying widely, frequent starts and stops render the mechanical load and thermal load change remarkably, leading to the extremely bad working environment, even a fault or stalling the engine. Accordingly we are supposed to make preliminary judgment on the fault phenomena timely, in an attempt to gain time for diagnosis and maintenancem.

Tugboat main engine fault system are as follows: ventilation system, fuel system, lubricating oil system, seawater system, freshwater system, starting air system, pressurization system. Then the fault phenomena having been analyzed and classified, a classification index system of tugboat main engine fault is initially built. The system classify faults with some certain index as shown in Figure 1.

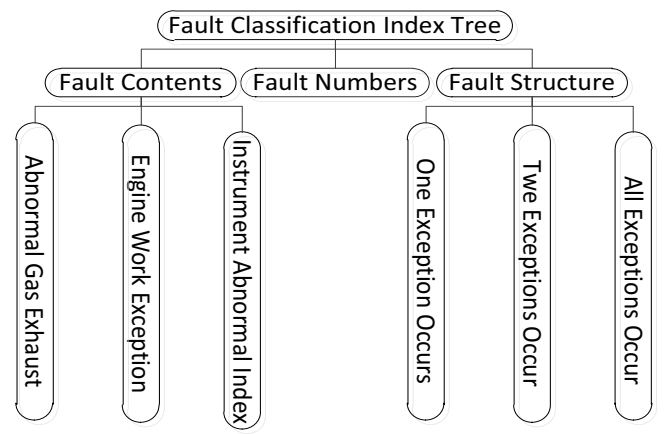

Figure 1. Fault Classification Index Tree.

\section{Fuzzy methods and procedures of fault classification $^{[2]}$}

Fault in tugboat main engine is a relative and qualitative fuzzy concept. It's difficult to have a quantitatively accurate represent of classification index as the contents are rich and formats are variable, which renders the 
classification a multi-factor and multi-level fuzzy classification.

A hierarchical factor-based fuzzy classification method is used in the classification, this is also to say, to classify from the bottom to the top, as well as from a single indicator to multi-indicator considered.

1). The classification index domain of the classification object is determined, then divided into $n$ subjects.

$$
U=\bigcup_{i=1}^{n} u_{i}
$$

where $u_{i}=\left\{u_{i 1}, u_{i 2}, \cdots, u_{i p_{i}}\right\}(i=1,2, \cdots, n)^{[3]}$

2). To each classification index subsets $u_{i}$, perform the single level and single indicator fuzzy transformation. Classification level domain $V=\left\{v_{1}, v_{2}, \cdots, v_{q}\right\}$ is given, where $q$ is better to be an odd number so that we can have a middle level.

Quantify each indicator in $u_{i}$, the membership of a certain fault to all categories classified by single indicator is determined. The value of membership function can be determined using expert scoring method. So we can have the single indicator fuzzy matrix $R_{i}$ of $u_{i}$.

The fuzzy weight vector of every indicator in $u_{i}$ is determined as

$$
A=\left(a_{i 1}, a_{i 2}, \cdots, a_{i p_{i}}\right)
$$

Before performing the synthesis process, $A_{i}$ needs to be normalized. Ensure

$$
\sum_{r=1}^{p_{i}} a_{i r}=1
$$

Performing fuzzy transformation to $A_{i}$ and $R_{i}$ gives us $B_{i}$, the single level classification result vector of, which is ${ }^{[3]}$

$$
\left\{\begin{array}{l}
A_{i} \cap R_{i}=B_{i} \\
B_{i}=\left(b_{i 1}, b_{i 2}, \cdots, b_{i m}\right)
\end{array}(i=1,2, \cdots, s)\right.
$$

The fuzzy weight vector of comprehensive index $u_{i}(i=1,2, \cdots, s)$ is given as follow

$$
A=\left(a_{1}, a_{2}, \cdots, a_{s}\right)^{[3]}
$$

3). Multi-level fuzzy classification. Repeat steps 1 2 using $u_{i}$ as the comprehensive index and $B_{i}$ as the single indicator classification result. We can have Multi-level fuzzy classification model.

4). Analysis of fuzzy classification results. The weighted average method is used to process fuzzy classification result vectors. The basic idea of this process is firstly to get the rank of classification result vector according to its position, which is to use $1,2, \cdots, q$ to represent every categories, and then secondly to get the weighted sum of each components' rank of result vector, thus we can have the relative position of classified object, written as

$$
E=\sum_{j=1}^{q} b_{j}^{k} j / \sum_{j=1}^{q} b_{j}^{k}
$$

where $k$ is a coefficient to be determined for controlling the import of larger $b_{j} \cdot k$ is often set as 1 or 2 , so we can sort several classified objects in the order of $E^{[3]}$.

\section{Key techniques in classification}

\subsection{Knowledge expression}

\subsubsection{Production rule}

Abnormal gas exhaust, engine work exception as well as instrumental abnormal index and the fault structure are identified and summarized as knowledge, based on the aforementioned classification index system. The knowledge is expressed as production rule with a basic structure below:

$$
\text { IF }<A>T H E N<B>
$$

In which, $\mathrm{A}$ is the production prerequisite, and $\mathrm{B}$ is a set of conclusions. Both of $\mathrm{A}$ and $\mathrm{B}$ can generously be expressions composed by text, numbers and logical operators AND, OR, NOT.

Here in our research, the prerequisite A led by IF of production rule is the classification index of different levels, and conclusion B led by THEN is the corresponding membership function.

\subsubsection{Frame structure}

Corresponding to the hierarchical structure of classification index tree, the multiple nested frame structure, such as \{Frame $\{$ Sub-Frame $\{$ Slot $\{$ Side $\{$ SubSide $\{$ Value... $\}\}\}\}\}\}$, is used for the knowledge expression to manage the production rules. The framework is composed by different levels. Its basic logical structure can be expressed as:

\{Frame $\{$ Slot 1 \{ Side 1 \{Value 1 ,

Value 2,

Value $\mathrm{m}\}$

Side 2 \{Value ...

Slot $2\{\ldots\}$

...

Slot $n\{\ldots\}$

\})

In this structure, location frame is for the classified object, such as a certain fault record. Slot is for the classification index, such as the fault content. Side is for classification sub-index, such as gas exhaust. Value is for classification indicator, such as exhaust pipe's blowing out black smoke. Compatible with the index, one side can have lots of value where production rules would be stored.

This frame-rule mixed expression of knowledge can greatly simplify the workload of the inference engine, 
reduce the search volume compared with a simple rule system, shorten the reasoning time and improve the efficiency of solving.

\subsection{Hierarchical structure of knowledge base}

A comprehensive knowledge base is necessary for the classification. Hierarchical tree structure, as shown in the Figure 2, is used to build to knowledge base, considering the contents and structure of classification index system[4].

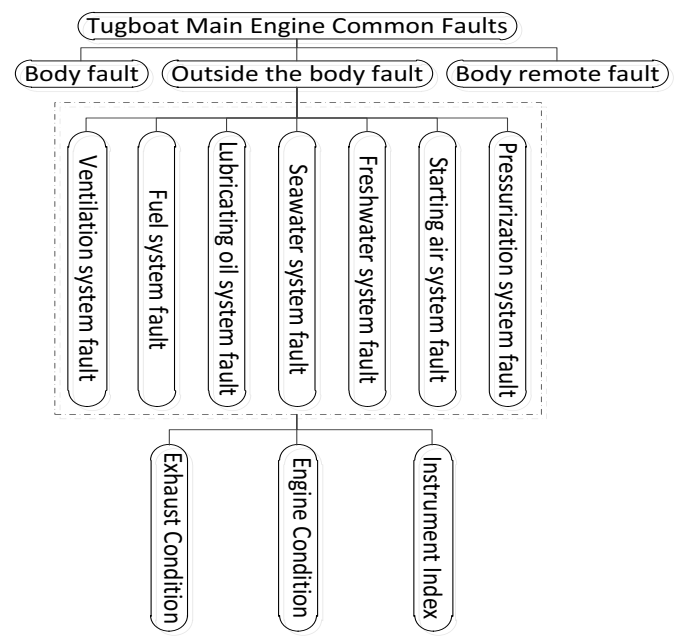

(a) Top level of knowledge base structure
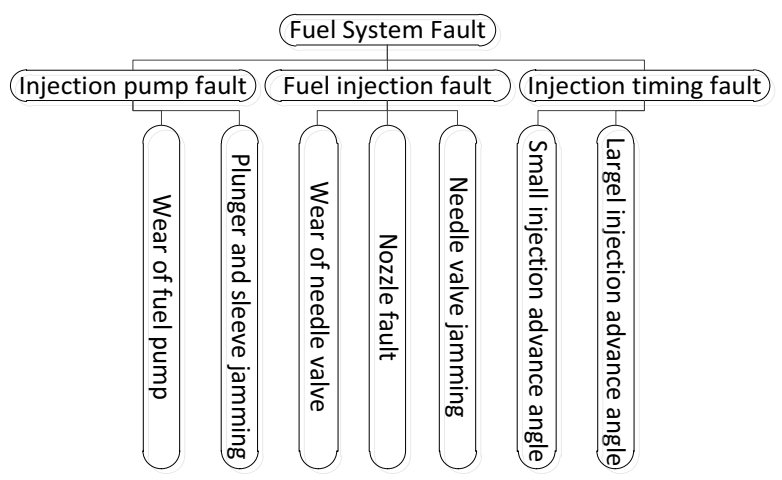

(b) Second level of knowledge base structure

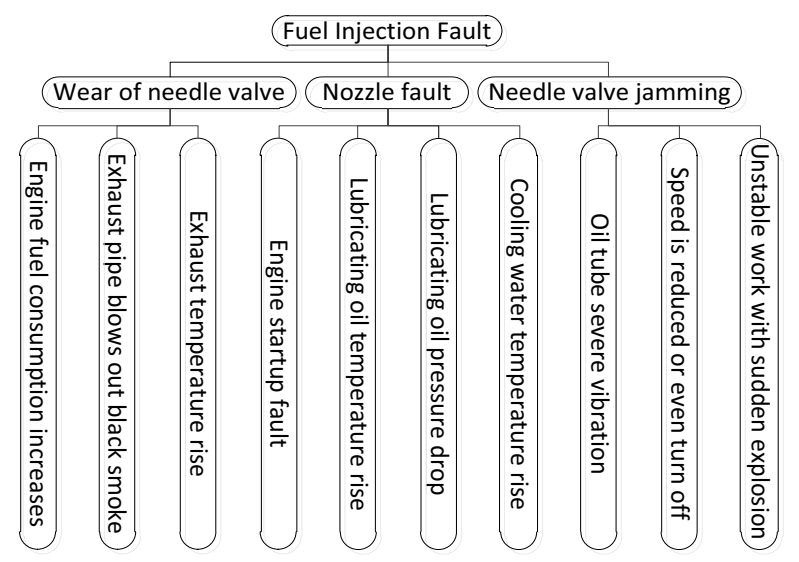

(c) Bottom level of knowledge base structure

Figure 2. Hierarchical Structure of the Knowledge Base.

The storage, management and maintain of knowledge can be easily achieved within this structure[5], through which the knowledge will also be conveniently used in inference.

\subsection{Pre-process and inference}

\subsubsection{Pre-processor}

Demanded by the classification knowledge, feature factors in a fault phenomenon must be extracted to compose the fact library. The pre-processor we used on our research is described as follows.

For a complete fault record, pre-processor firstly filtered the contents of features in it, such as many kinds of exhaust gas condition, engine condition, instrumental index, to compose the Feature Factor Sets. Then the number of all kinds of factors, which is structurally corresponding with the original text, is extracted after the filtering and counting. The results consist the fact library, and will be matched with the perquisite of rules.

A record is given here. A fault record in tugboat main engine we collected is "Exhaust pipe blows out black smoke and its temperature increases; unstable work with sudden explosion then power and speed decrease; at the same time, engine fuel consumption rises with oil tube of high temperature severe vibration; cooling water's temperature increases; temperature of lubricating oil rises and its pressure drops".

The information extract after pre-processing is listed in Table 1.

Table 1. The pre-processor result of a fault record.

\begin{tabular}{|c|c|c|}
\hline Content & Fault Name & Number \\
\hline \multirow{4}{*}{$\begin{array}{c}\text { Exhaust gas } \\
\text { condition }\end{array}$} & Blowing out black smoke & I \\
\cline { 2 - 3 } & Exhaust gas`s temperature rises & II \\
\hline \multirow{4}{*}{$\begin{array}{c}\text { Engine } \\
\text { condition }\end{array}$} & Engine power decreases & III \\
\cline { 2 - 3 } & $\begin{array}{c}\text { Unstable work with sudden } \\
\text { explosion }\end{array}$ & IV \\
\cline { 2 - 3 } & Oil tube`s severe vibration & V \\
\cline { 2 - 3 } & Engine fuel consumption rises & VI \\
\hline \multirow{4}{*}{$\begin{array}{c}\text { Instrumental } \\
\text { index }\end{array}$} & Lubricating oil`s temperature rises & VII \\
\cline { 2 - 3 } & Speed decreases & IX \\
\cline { 2 - 3 } & Lubricating oil`s pressure drops & X \\
\cline { 2 - 3 } & &
\end{tabular}

\subsubsection{Inference engine}

Fitted with the knowledge expression, forward inference engine is applied in our research. By matching the fact library with the prerequisite of rules, the values of membership function can be determined through the inference engine. 
Indicators in different levels of index system are filled in the hierarchical structure of knowledge base. The slot and side that will be used according to the levels of classification are firstly determined by the inference engine, and then the rules needed in solving are searched. Contents in fact library is matched with the prerequisite of the rules in the rule library one by one. The membership function contained in the conclusion of rules successfully matched will be stored as one of the row elements of membership matrix.

\section{Implementation}

The collected fault record in tugboat main engine are classified with the methods above. To space limitations, the fault record in 4.3 is given next as an example of the general classification.

Given the classification categories domain $V$.

$V=\{$ Ventilation system fault $(\mathrm{V})$, fuel system fault $(\mathrm{F})$, lubricating oil system (L), seawater system fault (S), freshwater system fault (W), starting air system fault (A), pressurization system fault $(\mathrm{P})\}$

Main indicators are chosen, according to the contents referred, to build classification indicators domain $U$. The general indicators domain used in classification of different fault record is transcendental, while different kinds of sub-sets of indicators can also be generally used in classification of the same type of faults. The classification result is shown in Table 2. In "categories". I represents "Blowing out black smoke" while II is on the behalf of "Exhasut gas's temperature rises" and the rest are inferred in Table 1.

Table 2. The classification result of a fault record.

\begin{tabular}{|c|c|c|c|c|c|c|c|}
\hline $\begin{array}{c}\text { Categorie } \\
\text { s }\end{array}$ & $\mathbf{V}$ & $\mathbf{F}$ & $\mathbf{L}$ & $\mathbf{S}$ & $\mathbf{W}$ & $\mathbf{A}$ & $\mathbf{P}$ \\
\hline I & 0.22 & 0.62 & 0.02 & 0.03 & 0.04 & 0.01 & 0.06 \\
\hline II & 0.18 & 0.52 & 0.04 & 0.02 & 0.03 & 0.02 & 0.19 \\
\hline III & 0.22 & 0.31 & 0.05 & 0.03 & 0.21 & 0.02 & 0.16 \\
\hline IV & 0.23 & 0.49 & 0.05 & 0.02 & 0.02 & 0.02 & 0.17 \\
\hline V & 0.15 & 0.63 & 0.04 & 0.06 & 0.09 & 0.01 & 0.02 \\
\hline VI & 0.23 & 0.39 & 0.06 & 0.13 & 0.16 & 0.01 & 0.02 \\
\hline VII & 0.21 & 0.24 & 0.02 & 0.21 & 0.27 & 0.02 & 0.03 \\
\hline VIII & 0.03 & 0.39 & 0.34 & 0.02 & 0.03 & 0.01 & 0.18 \\
\hline IX & 0.21 & 0.32 & 0.04 & 0.03 & 0.21 & 0.03 & 0.16 \\
\hline X & 0.03 & 0.42 & 0.35 & 0.01 & 0.02 & 0.02 & 0.15 \\
\hline
\end{tabular}

However, when data in Table 2 are used as the fuzzy matrix $R$, the value of each component of weight vector is too small to affect the classification results since there are too many categories. So we divide the data into 3 groups(the first two as a group, III、 IV V V and VI as a group, the last four as the third group) . Fuzzy transformation is performed respectively to have

$$
R=\left[\begin{array}{lllllll}
0.20 & 0.57 & 0.03 & 0.02 & 0.04 & 0.02 & 0.12 \\
0.21 & 0.46 & 0.05 & 0.06 & 0.12 & 0.01 & 0.09 \\
0.12 & 0.35 & 0.18 & 0.07 & 0.13 & 0.02 & 0.13
\end{array}\right]
$$

Delphi method is used to determine the weights of each indicators. Here, we use the weight vector $A=(0.21$, $0.43,0.36$ )

Fuzzy transformation is performed to have $B=(0.21$, $0.43,0.18,0.07,0.13,0.02,0.13$ )

Weighted average method is used to analysis and process the fuzzy classification results. Set the value of each components of the resulting vector according to their positions, which means that, 1 for ventilation sysytem fault, 2 for fuel system fault, 3 for lubricating oil sysytem fault, 4 for seawater system fault, 5 for freshwater system fault, 6 for starting air system fault, 7 for pressurization system fault. The final classification results is calculated as Equation $(4)(k=2, q=7)$.

The calculation result is $E \approx 2.45$, which shows that it is between category 2 and 3 , and slightly partial to the second one. Thus it is probably a fuel system fault[6] or a lubricating oil system fault, though more likely to be the former. However, it can easily identified literally to both of those two categories. As for which one it actually belonged to, these two systems should be checked to determine the factual fault location. Nevertheless, in terms of the phenomenon of the fault record, it is difficult to distinguish whether it is a fuel system fault or a lubricating oil system fault. Therefore, this classification result is reasonable.

\section{Implementation}

With method and procedure above, a fault record in tugboat is classified for certification, and the result is considered reasonable and useful while the method and implementation are effective and efficient. Result shows that the classification index system, the method and the implementation technique can not only satisfy the actual need of the tugboat main engine fault classification, and also enable personnel to eliminate the fault as soon as possible, to gain time for the normal operation of tugboat.

\section{References}

1. Tikun Wang: Tugboat main engine fault diagnosis method based on the fault tree analysis (Master of Engineering, Dalian Maritime University, China 2012)

2. Xiaohui $\mathrm{Xu}$, Bingshu Tong. Fuzzy evaluation and implementation techniques of product structure design for assembly. Agricultural Machinery. 38,2 (2007) , p. 129-133 
3. Zhongxiong He :Fuzzy mathematics and applications(Tianjin science and technology press,China, 1982)

4. Qingmin Zhong: Experimental research on monitoring and diagnosing for diesel engine(Master of Science, Wuhan University of Technology, China 2008)

5. Yanyou Chai: Research on fault diagnosis of marine diesel engine based on kernel-based learning
theory(Doctor of Engineering, Harbin Engineering University, China 2012)

6. Xiaojun Ma, Zhi Wang, Chunguang Xing. Research on fault diagnosis for fuel injector system of marine diesel engine. Ship science and technology. 30,6 (2008) , p. 73-75,103 